\title{
Downregulation of FSTL-1 attenuates the inflammation injury during Streptococcus pneumoniae infection by inhibiting the NLRP3 and TLR4/NF-кB signaling pathway
}

\author{
LIANG CHEN $^{1}$ and ZHENSHE LIU ${ }^{2}$ \\ ${ }^{1}$ Department of Infectious Disease, Beijing Jishuitan Hospital, 4th Medical College of Peking University, Beijing 100096; \\ ${ }^{2}$ Department of Laboratory, Xian Yang Central Hospital, Xianyang, Shaanxi 712000, P.R. China
}

Received May 15, 2019; Accepted September 5, 2019

DOI: $10.3892 / \mathrm{mmr} .2019 .10752$

\begin{abstract}
Streptococcus pneumoniae-induced pneumonia is a common disease and major cause of community-acquired pneumonia. Previous studies have shown that Follistatin-like protein 1 (FSTL-1) serves important roles in regulating the inflammatory response. The present study aimed to investigate the effect of FSTL-1 on the inflammatory response during $S$. pneumoniae infection using in vitro and in vivo models. ELISAs were used to detect the production of interleukin (IL)-1 $\beta$, tumor necrosis factor- $\alpha$ and IL-6. Western blotting and reverse transcription-quantitative PCR were performed to determine the protein and mRNA expression of these factors. The results of the present study indicated that $S$. pneumoniae infection triggered a strong proinflammatory response and a high level of FSTL-1 expression in mouse bone marrow-derived macrophages. Moreover, FSTL-1 may be required for the production of inflammatory factors during $S$. pneumoniae infection by regulating nucleotide oligomerization domain-like receptor protein 3 in vitro and in vivo. In addition, it was found that the Toll-like receptor $4 /$ nuclear factor- $\kappa \mathrm{B}$ signaling pathway was involved in the inflammatory response regulated by FSTL-1. The findings of the present study suggested that FSTL-1 plays an important role in the inflammatory response during $S$. pneumoniae infection, providing a potential therapeutic target for reducing morbidity and mortality in patients with pneumonia.
\end{abstract}

Correspondence to: Dr Zhenshe Liu, Department of Laboratory, Xian Yang Central Hospital, 78 East of Renming Road, Weicheng, Xianyang, Shaanxi 712000, P.R. China

E-mail: zhensheliu1111@126.com

Key words: Streptococcus pneumoniae, Follistatin-like protein 1, nucleotide oligomerization domain-like receptor protein 3 , Toll-like receptor $4 / \mathrm{NF}-\kappa \mathrm{B}$, inflammation

\section{Introduction}

Bacterial pneumonia, including Streptococcus pneumoniae, Staphylococcus aureus, Klebsiella pneumoniae, Haemophilus influenzae and Pseudomonas aeruginosa, is the most common form of pneumonia and one of the most common infectious diseases, and poses a threat to the health of children and the elderly (1). Among all bacteria, S. pneumoniae is the most common cause of pneumonia (2). Although antibiotics are the main treatment for pneumonia, the antimicrobial resistance of S. pneumoniae pneumonia has increased significantly over the past few decades (3). Therefore, there is concern regarding $S$. pneumoniae pneumonia $(4,5)$. The diseases caused by S. pneumoniae can be partially attributed to the inflammatory response during infection; however, research into the inflammatory injury caused by $S$. pneumoniae pneumonia is required.

According to a previous study, in the early stage of pneumonia, the phenotype of macrophages transforms and they release a large number of proinflammatory cy tokines, including tumor necrosis factor- $\alpha$ (TNF- $\alpha$ ), interleukin (IL)- 6 and IL-8, the pathogen invades the alveoli, leading to an imbalance of the inflammatory response and to acute lung injury (6). The occurrence and development of pneumonia is related to the imbalance of the inflammatory response (7-9). Follistatin-like protein 1 (FSTL-1) is a secretory glycoprotein produced mainly by cells of the mesenchymal lineage, including cardiomyocytes, chondrocytes, fibroblasts and endotheliocytes, which regulate various biological functions, such as cell proliferation, differentiation, apoptosis and metabolism (10). A previous study showed that FSTL-1 serves as a proinflammatory protein that plays important roles in the inflammatory response (11). Increased levels of FSTL-1 in macrophages lead to the upregulation of proinflammatory factors, including IL-1 $\beta$, TNF- $\alpha$ and IL-6 (12), supporting a role for FSTL-1 in inflammation. Furthermore, Chaly et al (13) found that FSTL-1 modulated the proinflammatory factor IL-1 $\beta$ by activating nucleotide oligomerization domain-like receptor protein 3 (NLRP3) in monocytes and macrophages. The activation of NLRP3 recruits apoptosis-associated spot-like protein (ASC) and pro-caspase-1, converting inactive pro-caspase- 1 into active caspase-1, promoting the maturation of pro-IL-1 $\beta$ and 
pro-IL-18, which in turn activate caspase-1 through autocrine or paracrine signaling to release more mature IL-1 $\beta(14,15)$. The persistence of IL-1 $\beta$ amplifies the inflammatory response, leading to inflammation damage in normal tissues (16). Therefore, the present study was designed to understand the molecular mechanism of FSTL-1, and determine the relationship between FSTL-1 and NLRP3 in inflammation injury during $S$. pneumoniae infection.

Toll-like receptors (TLRs) serve important roles in preventing pathogen invasion by detecting pathogen-associated molecular patterns (PAMPs) (17). In innate immune cells, TLRs initiate complex signaling pathways by recognizing PAMPs, leading to the activation of several transcription factors, including NF- $\mathrm{B}$ and activator protein-1, which are involved in the production of proinflammatory cytokines, chemokines and the induction of various regulatory genes (18). In the present study, whether the TLR4/NF- $\kappa \mathrm{B}$ signaling pathway is involved in the inflammatory response regulated by FSTL-1 during $S$. pneumoniae infection was investigated.

In this study, the functional role of FSTL-1 in vitro and in vivo after $S$. pneumoniae infection was examined. The results indicated that downregulation of FSTL-1 suppressed inflammation injury in bone marrow-derived macrophages (BMDMs) after $S$. pneumoniae infection through the NLRP3 and TLR4/NF- $\kappa \mathrm{B}$ signaling pathways.

\section{Materials and methods}

Bacterial culture. S. pneumoniae (ATCC 6305; Bena Culture Collection) was cultured in brain heart infusion broth (BD Biosciences) under anaerobic conditions $\left(95 \% \quad \mathrm{~N}_{2} / 5 \% \mathrm{CO}_{2}\right)$ overnight at $37^{\circ} \mathrm{C}$. Dilution of samples in brain heart infusion broth was used to quantify colony forming units (CFU), and colony counts $\mathrm{x}$ dilution was used to determine titers.

Animals. A total of 25 (11 male and 14 female) wild type (WT) and 25 (11 male and 14 female) FSTL-1 knockout (KO) C57BL/6 mice (8-12 weeks old, 22-25 g) were provided by CasGene Biotech Co., Ltd (Beijing, China). The mice were fed on standard pellet chow and water ad libitum and housed in a controlled SPF-class environment with 12-h light/dark cycle at an ambient temperature of $24 \pm 1{ }^{\circ} \mathrm{C}$ and a humidity of $55 \pm 5 \%$. The present study was conducted according to Xian Yang Central Hospital medical guidelines and the experiment was approved by Xian Yang Central Hospital Ethics Committee.

S. pneumoniae infection. The mice were anesthetized with $2 \%$ isoflurane in $\mathrm{O}_{2}$ using a face mask for $5 \mathrm{~min}$ and were then intranasally inoculated with $1 \times 10^{3} \mathrm{CFU}$ of $S$. pneumoniae (50 $\mu 1$ in PBS) once when they were fully sedated. The uninfected wild-type (WT) mice and FSTL-1 KO mice were treated with only $50 \mu \mathrm{l}$ of PBS. The animals were euthanized and samples were collected for further analysis 7 days after infection.

Cell culture. BMDMs were obtained from the bone marrow of the femur and tibia of the C57BL/6 mice as previously described (19). Bone marrow cells were added to L929 media containing DMEM(Life Technology; Thermo Fisher Scientific, Inc.), containing $40 \mathrm{ng} / \mathrm{ml}$ macrophage colony-stimulating factor (Life Technology; Thermo Fisher Scientific, Inc.), 10\% FBS (Life Technology; Thermo Fisher Scientific, Inc.), $1 \%$ non-essential amino acids (Life Technology; Thermo Fisher Scientific, Inc.) and 1\% cyanomycin (Life Technology; Thermo Fisher Scientific, Inc.). The cells were cultured for 2 days at $37^{\circ} \mathrm{C}$ with $5 \% \mathrm{CO}_{2}$ and the bone marrow cells differentiated into macrophages. The BMDMs were washed three times with PBS and cultured in DMEM containing 10\% FBS. BMDMs were collected 0, 4, 12, 24 and $36 \mathrm{~h}$ after infection with $S$. pneumoniae (50 CFU) or media alone for RT-qPCR, western blotting and ELISA analysis.

Cell transfection. Lentiviral-based scrambled-short hairpin (sh)RNA vectors, FSTL-1-shRNA vectors, pcDNA3.1-FSTL-1-overexpression (FSTL-1-OE) vectors, FSTL-1-control vectors and NLRP3-shRNA vectors were synthesized by Shanghai GeneChem Co., Ltd. FSTL-1-shRNA and FSTL-1-OE vectors were used to knockdown or overexpress FSTL-1, respectively. NLRP3-shRNA vectors were used to knockdown NLRP3. Full-length FSTL-1 cDNA was cloned into pcDNA3.1 plasmid and the shRNAs targeting FSTL-1 or NLRP3 was inserted into pLKO.1 plasmid. The target sequence of FSTL-1 shRNA was CTTCCTCGGAGC GTGGTGA, NLRP3 shRNA was CTCGGTCCAACATCT AGCA. Then, $5 \times 10^{5}$ cells/well BMDMs were infected with a mixture of $40 \mathrm{nmol} / \mathrm{ml} \mathrm{shRNA}$ or $50 \mathrm{nmol} / \mathrm{ml} \mathrm{pcDNA3.1} \mathrm{and}$ the transfection was performed using Lipofectamine $2000^{\circledR}$ reagent (Invitrogen; Thermo Fisher Scientific, Inc.) following manufacturer's instructions for 3 days.

Sample collection and analysis. The bronchoalveolar lavage fluids (BALFs) and lung tissues of the mice were collected at 7 days post-infection ( $n=6 /$ group). BALF was harvested using $1.5 \mathrm{ml}$ PBS supplemented with complete protease inhibitor cocktail (Roche Diagnostics). BALFs were isolated via centrifugation at $1,000 \times \mathrm{g}$ for $10 \mathrm{~min}$ at $4^{\circ} \mathrm{C}$ and then stored at $-80^{\circ} \mathrm{C}$ for further analysis. The fresh lung tissues collected from mice with different treatments were immediately snap-frozen in liquid nitrogen and stored at $-80^{\circ} \mathrm{C}$ until use.

Reverse transcription-quantitative $(R T-q) P C R$. Total RNA was extracted from BMDMs using TRIzol reagent (Invitrogen; Thermo Fisher Scientific, Inc.), according to the manufacturer's protocol. Total RNA was quantified using a NanoDrop $^{\mathrm{TM}} 2000$ (NanoDrop Technologies; Thermo Fisher Scientific, Inc.). Complementary DNA was synthesized using the M-MLV Reverse Transcriptase kit (Invitrogen; Thermo Fisher scientific, Inc.). The RT conditions were the following: $5 \mathrm{~min}$ at $25^{\circ} \mathrm{C}, 30 \mathrm{~min}$ at $42^{\circ} \mathrm{C}$ and $5 \mathrm{~min}$ at $85^{\circ} \mathrm{C}$. The quantification of all gene transcripts was performed using an SYBR Green II real-time PCR kit (Takara Bio, Inc.). The thermocycling conditions: Initial denaturation at $95^{\circ} \mathrm{C}$ for $3 \mathrm{~min}$, followed by 40 cycles of $95^{\circ} \mathrm{C}$ for $15 \mathrm{sec}$ and $60^{\circ} \mathrm{C}$ for $60 \mathrm{sec}$. $\beta$-actin was used as an internal control. Each experiment was run in triplicate. The $2^{-\Delta \Delta C q}$ method (20) was used calculate the relative expression of genes. The following primers were used: FSTL-1, forward 5'-TTATGATGGGCACGCAAAGAA-3', reverse 5'-ACTGCCTTTAGAGAACCAGCC-3'; NLRP3, forward 5'-CCAGACACTCATGTTGCCTGTTC-3', reverse 5'-GAGGCTCCGGTTGGTGCTTA-3'; and $\beta$-actin, forward 
A

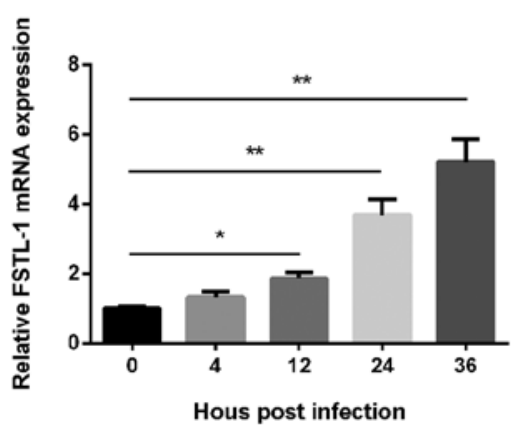

B

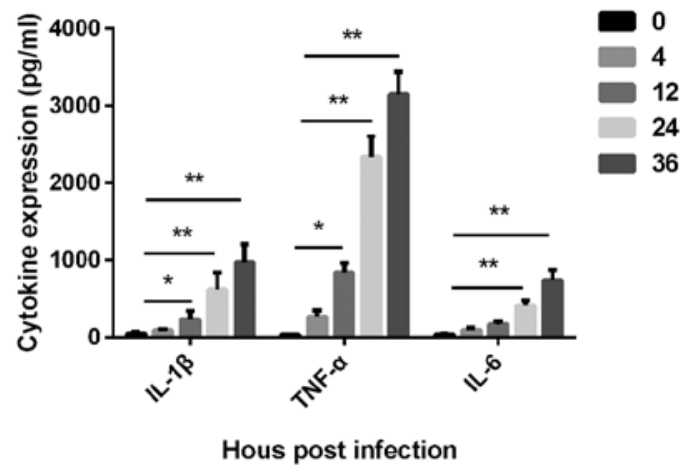

C
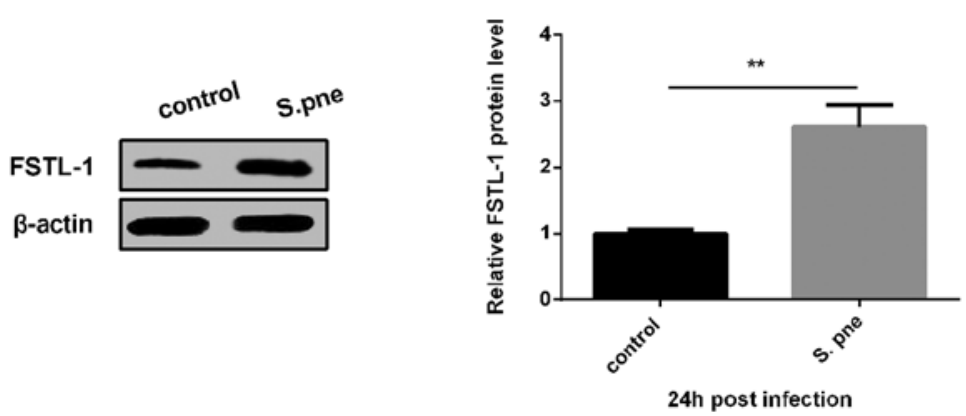

Figure 1. Increased expression of FSTL-1 in BMDMs infected with $S$. pneumoniae. (A) FSTL-1 mRNA expression was determined in BMDMs following $S$. pneumoniae infection for $0,4,12,24$ and $36 \mathrm{~h}$ via reverse transcription-quantitative PCR analysis. (B) IL-1 $\beta$, TNF- $\alpha$ and IL- 6 expression levels were determined in BMDMs following S. pneumoniae infection for 0, 4, 12, 24 and $36 \mathrm{~h}$ using ELISAs. (C) FSTL-1 protein levels were determined in BMDMs following $S$. pneumoniae infection for $24 \mathrm{~h}$ via western blotting. Similar results were obtained from at least three independent experiments. Data are expressed as the mean $\pm \mathrm{SD} .{ }^{*} \mathrm{P}<0.05,{ }^{* *} \mathrm{P}<0.01$ vs. $0 \mathrm{~h}$ or control. BMDMs, bone marrow-derived macrophages; FSTL-1, Follistatin-like protein 1; IL, interleukin; S.pne, Streptococcus pneumoniae; TNF- $\alpha$, tumor necrosis factor- $\alpha$.

\section{5'-CCTCATGAAGATCCTGACCG-3' and reverse 5'-ACC GCTCATTGCCGATAGTG-3'.}

ELISAs. TNF- $\alpha$ (cat. no. PT512), IL-6 (cat. no. PI326) and IL-1 $\beta$ (cat. no. PI301) ELISA kits were purchased from Beyotime Institute of Biotechnology. The production of TNF- $\alpha$, IL-6 and IL- $1 \beta$ in the supernatants of BMDMs and BALFs of mice following different treatments was detected using the ELISA kits according to the manufacturer's protocols. A microplate reader was used to measure the optical density of each group at $450 \mathrm{~nm}$.

Western blotting. The proteins were extracted from BMDMs and lung tissues with the use of radioimmunoprecipitation assay buffer (Beijing Solarbio Science \& Technology Co., Ltd.), and the protein concentration was measured using a BCA Protein assay kit (Thermo Fisher Scientific, Inc.). Subsequently, an equal amount of protein $(50 \mu \mathrm{g})$ was separated via $10-12 \%$ SDS-PAGE and transferred to nitrocellulose membranes. After blocking with $5 \%$ skim milk for $2 \mathrm{~h}$ at room temperature, the membranes were incubated with primary rabbit anti-FSTL-1 (1:500; Abcam; cat. no. ab71548), anti-TLR4 (1:500; Abcam; cat. no. ab13867), rabbit anti-NLRP3 (1:1,000; Abcam; cat. no. ab214185), rabbit anti-ASC (1:1,000; Cell Signaling Technology, Inc.; cat. no. 67824), rabbit anti-cleaved-caspase-1 (1:1,000; Cell Signaling Technology, Inc.; cat. no. 89332), rabbit anti-phosphorylated (p)-NF- $\kappa$ B p65 (1:1,000; Cell Signaling Technology, Inc.; cat. no. 3033), rabbit anti-NF-кB p65 (1:1,000; Cell Signaling Technology, Inc.; cat. no. 242), rabbit anti-p-NF- $\kappa \mathrm{B}$ inhibitor $\alpha(\mathrm{p}-\mathrm{I} \kappa \mathrm{B} \alpha ; 1: 1,000$; Cell Signaling
Technology, Inc.; cat. no. 5209), rabbit anti-IкB $\alpha(1: 1,000$; Cell Signaling Technology, Inc.; cat. no. 4812) and mouse anti- $\beta$-actin (1:1,000; Abcam; cat. no. ab8226) overnight at $4^{\circ} \mathrm{C}$. Subsequently, the membranes were incubated with horseradish peroxidase (HRP)-conjugated secondary antibodies (goat anti-rabbit IgG-HRP; 1:2,000; cat. no. sc-2004; goat anti-mouse IgG-HRP; 1:2,000; cat. no. sc-2005; Santa Cruz Biotechnology, Inc.) for $2 \mathrm{~h}$ at temperature. An ECL kit (GE Healthcare) was then applied to detect the signals. The gray value analysis of target band was analyzed by Image $\mathbf{J}$ software 1.48u (National Institutes of Health). $\beta$-actin was used as a loading control.

Statistical analysis. Data are presented as the mean \pm SD from at least three independent experiments. Student's t-test was used for comparisons between two groups and ANOVA followed by Tukey's post-hoc test was used when there were more than two groups to calculate significance among the different groups. Statistical analyses were performed using SPSS 17.0 Software (SPSS, Inc.). $\mathrm{P}<0.05$ was considered to indicate a statistically significant difference.

\section{Results}

FSTL-1 is upregulated in BMDMs after S. pneumoniae injection. To investigate the functional role of FSTL-1 in $S$. pneumoniae infection, the mRNA expression of FSTL1 in BMDMs was determined following $S$. pneumoniae infection for $0,4,12,24$ and $36 \mathrm{~h}$ via RT-qPCR analysis. As shown in Fig. 1A, FSTL-1 mRNA expression was significantly elevated 
A

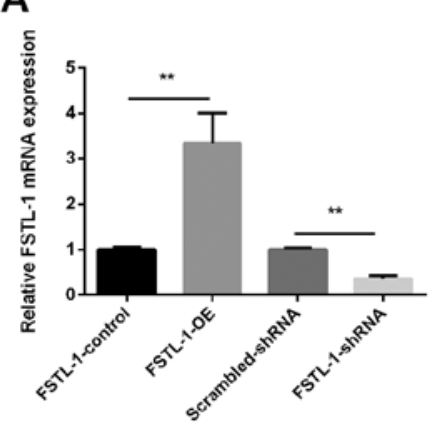

C

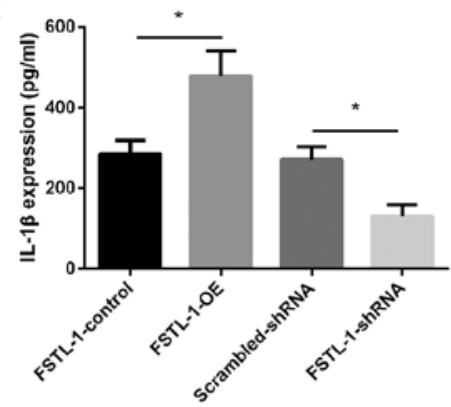

B

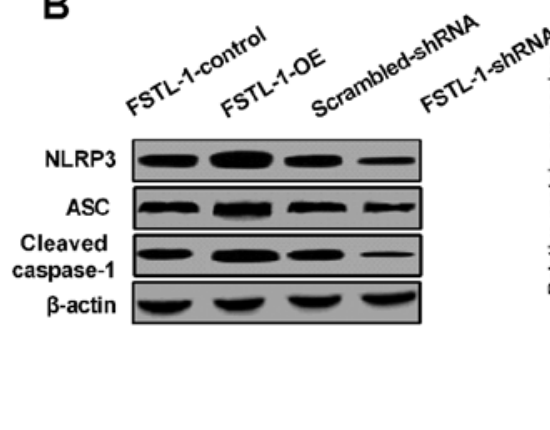

D

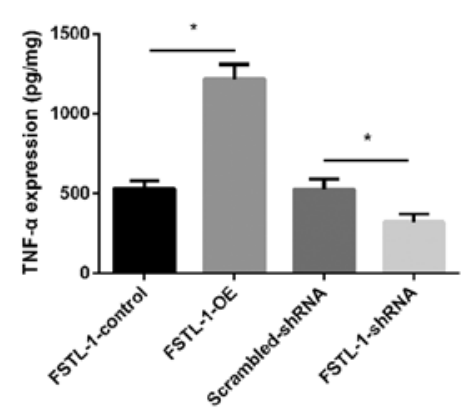

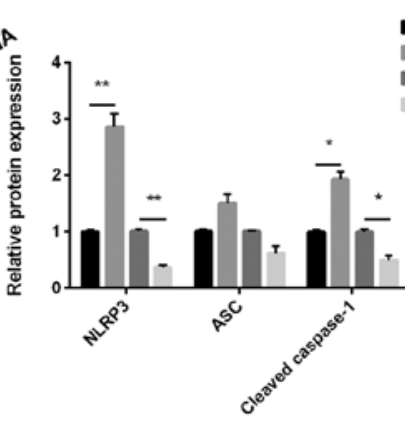

E

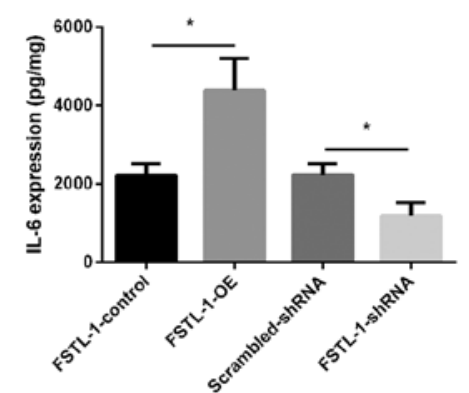

Figure 2. FSTL-1 promotes the production of inflammatory cytokines during S. pneumoniae infection in BMDMs by regulating NLRP3. (A) Relative expression of FSTL-1 determined via reverse transcription-quantitative PCR analysis in BMDMs after FSTL-1 OE or shRNA. (B) NLRP3, ASC and cleaved caspase-1 protein levels were determined in BMDMs using western blotting after FSTL-1 OE or shRNA. (C) IL-1 $\beta$, (D) TNF- $\alpha$ and (E) IL-6 expression levels were determined in BMDMs using ELISA after FSTL-1 OE or shRNA. Similar results were obtained from at least three independent experiments. Data are expressed as the mean \pm SD. ${ }^{*} \mathrm{P}<0.05,{ }^{* *} \mathrm{P}<0.01$ vs. respective control. BMDMs, bone marrow-derived macrophages; FSTL-1, Follistatin-like protein 1 ; NLRP3, nucleotide oligomerization domain-like receptor protein 3; OE, overexpression; shRNA, short hairpin RNA; ASC, apoptosis-associated spot-like protein; IL, interleukin; S. pneumoniae, Streptococcus pneumoniae; TNF- $\alpha$, tumor necrosis factor- $\alpha$.

in BMDMs after $S$. pneumoniae injection. The production of the inflammatory cytokines IL- $1 \beta$, TNF- $\alpha$ and IL- 6 was also increased after $S$. pneumoniae injection as detected using ELISAs (Fig. 1B). FSTL-1, IL-1 $\beta$, TNF- $\alpha$ and IL-6 levels were increased in a dose-dependent manner and exhibited a significant difference at $24 \mathrm{~h}$ post-infection compared with $0 \mathrm{~h}$. Therefore, cells were collected $24 \mathrm{~h}$ after infection for further study. The protein level of FSTL-1 in BMDMs was determined following $S$. pneumoniae infection for $24 \mathrm{~h}$. The results indicated that FSTL-1 was significantly increased (Fig. 1C). These findings suggested that FSTL-1 may play an important role in the inflammatory response during S. pneumoniae infection.

FSTL-1 modulates inflammatory injury during S. pneumoniae infection via NLRP3 in vitro. To investigate the potential mechanism of FSTL-1 against inflammatory injury during S. pneumoniae infection in BMDMs, FSTL-1 expression was increased or decreased using FSTL-1-OE or FSTL-1-shRNA vectors, respectively, in BMDMs. FSTL-1 expression was significantly increased in the FSTL-1-OE group, whereas it was decreased in the FSTL-1-shRNA group (Fig. 2A). Western blotting results indicated that NLRP3 and cleaved caspase-1 levels were increased significantly following the overexpression of FSTL-1, while they were decreased after the depletion of FSTL-1 in BMDMs infected with S. pneumoniae. However, ASC expression was not significantly affected by changes in FSTL-1 expression (Fig. 2B). ELISA results showed that increasing FSTL-1 levels enhanced, whereas decreasing FSTL-1 inhibited, the production of IL-1 $\beta$, TNF- $\alpha$ and IL-6
(Fig. 2C-E). These data indicated that the downregulation of FSTL-1 may attenuate inflammatory injury by inhibiting NLRP3.

Downregulation of NLRP3 inhibits inflammatory injury during $S$. pneumoniae infection in vitro. To investigate the role of NLRP3 in inflammatory injury during $S$. pneumoniae infection, NLRP3 levels were determined in BMDMs. As shown in Fig. 3A, NLRP3 was significantly increased in BMDMs after $S$. pneumoniae infection. NLRP3 mRNA and protein expression levels were decreased significantly by NLRP3-shRNA, as shown in Fig. 3B and C. ELISA results showed that decreasing NLRP3 expression inhibited the expression of IL-1 $\beta$, TNF- $\alpha$ and IL- 6 in BMDMs after S. pneumoniae infection (Fig. 3D-F). These data demonstrated that the downregulation of NLRP3 inhibited inflammatory injury during $S$. pneumoniae infection.

TLR4/NF- $\kappa B$ signaling pathway is involved in inflammatory injury during $S$. pneumoniae infection and is regulated by FSTL-1 in vitro. Western blotting was used to determine the protein level of TLR4 and the downstream targets of the NF- $\kappa$ B pathway. The results showed that TLR4 expression was increased by FSTL-1-OE, while it was decreased by FSTL-1-shRNA (Fig. 4A and B). The phosphorylation of $\mathrm{NF}-\kappa \mathrm{B}$ p 65 and $\mathrm{I} \kappa \mathrm{B} \alpha$ was activated by FSTL-1-OE and was inactivated by FSTL-1-shRNA. These data suggested that TLR4/NF- $\kappa$ B signaling was involved in inflammatory injury during $S$. pneumoniae infection regulated by FSTL-1 in vitro. 
A

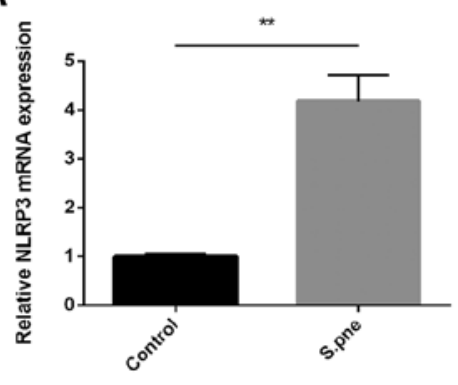

D

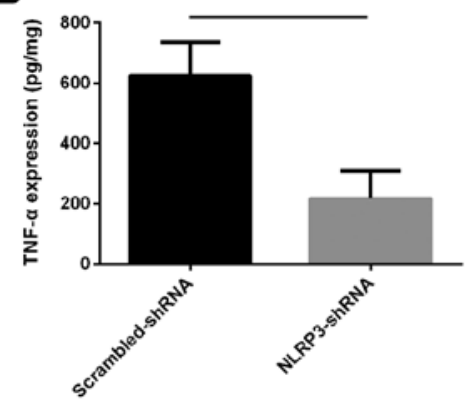

B

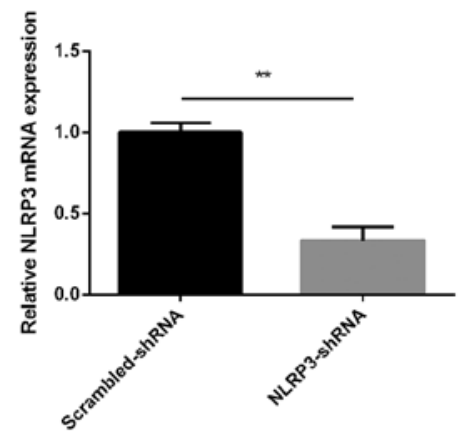

E

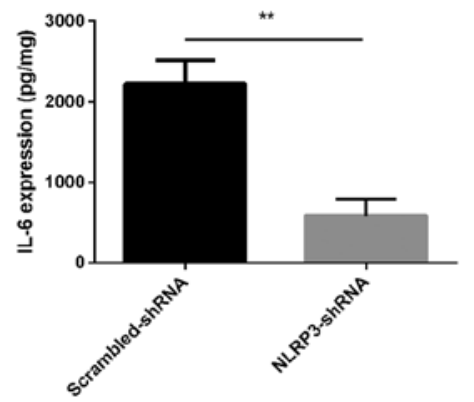

C

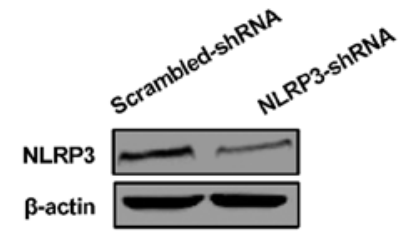

$\mathbf{F}$

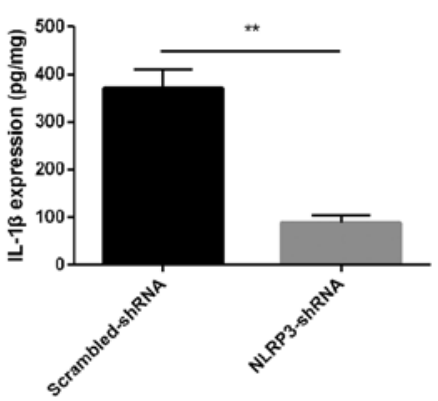

Figure 3. Downregulation of NLRP3 inhibits the production of inflammatory cytokines during S. pneumoniae infection in BMDMs. (A) NLRP3 mRNA expression was determined in BMDMs infected with S. pneumoniae via RT-qPCR analysis. Relative (B) mRNA and (C) protein expression of NLRP3 as determined using RT-qPCR or western blot analyses, respectively, in BMDMs after depletion of NLRP3. (D) TNF- $\alpha$, (E) IL-6, and (F) IL-1 $\beta$ expression was determined in BMDMs using ELISAs after the depletion of NLRP3. Similar results were obtained from at least three independent experiments. Data are expressed as the mean $\pm \mathrm{SD}$. ${ }^{*} \mathrm{P}<0.05,{ }^{* *} \mathrm{P}<0.01$. BMDMs, bone marrow-derived macrophages; NLRP3, nucleotide oligomerization domain-like receptor protein 3; shRNA, short hairpin RNA; IL, interleukin; S.pne, Streptococcus pneumoniae; TNF- $\alpha$, tumor necrosis factor- $\alpha$; RT-qPCR, reverse transcription-quantitative PCR.

A

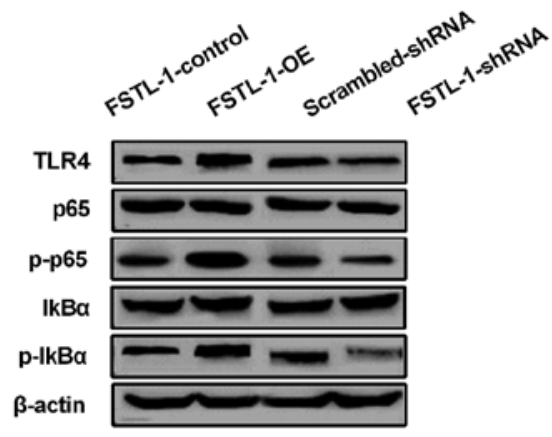

B
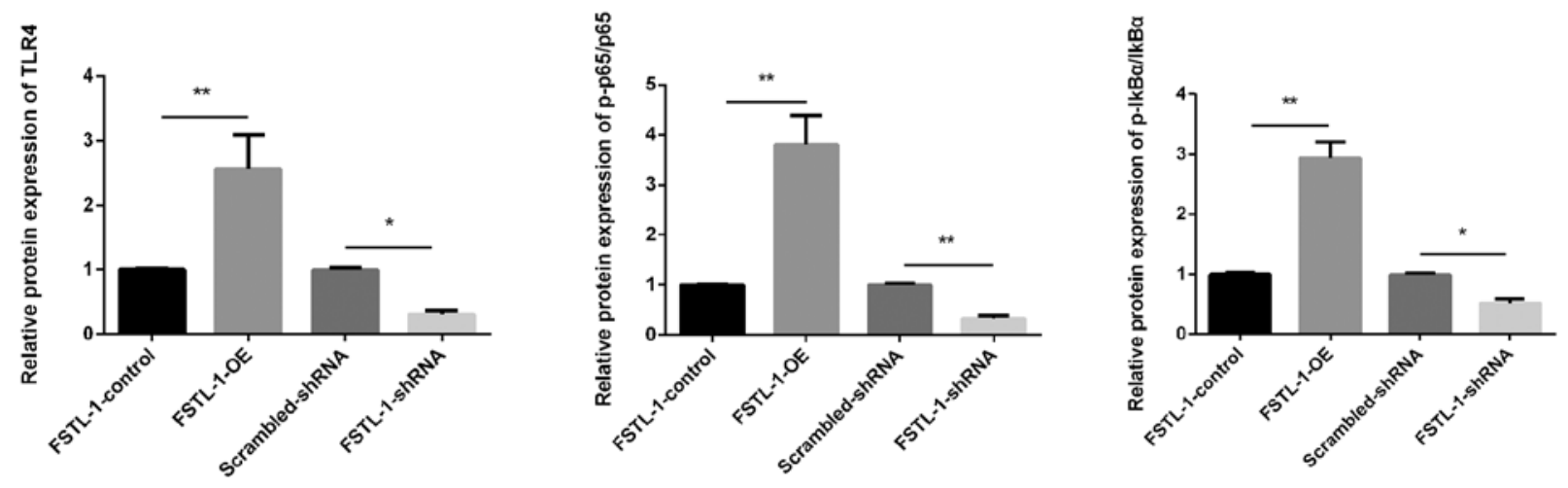

Figure 4. TLR4/NF- $\mathrm{KB}$ signaling is involved in inflammatory injury during $S$. pneumoniae infection and is regulated by FSTL-1. (A) Levels of TLR4, NF- $\mathrm{BB}$ p65, p-p65, IкB $\alpha, \mathrm{p}-\mathrm{I} \kappa \mathrm{B} \alpha$ protein levels determined using western blotting in BMDMs after transfection with FSTL-1-OE or FSTL-1-shRNA. (B) Relative protein levels of TLR4, p-p65/p65, p-I $\kappa \mathrm{B} \alpha / \mathrm{I} \kappa \mathrm{B} \alpha$ were quantified in BMDMs after transfection with FSTL-1-OE or FSTL-1-shRNA. Similar results were obtained from at least three independent experiments. Data are expressed as the mean $\pm \mathrm{SD}$. " $\mathrm{P}<0.05,{ }^{* *} \mathrm{P}<0.01 \mathrm{vs}$. FSTL-1 control. BMDMs, bone marrow-derived macrophages; FSTL-1, Follistatin-like protein 1; S.pne, Streptococcus pneumoniae; TLR4, Toll-like receptor 4; OE, overexpression; shRNA, short hairpin

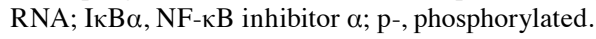


A

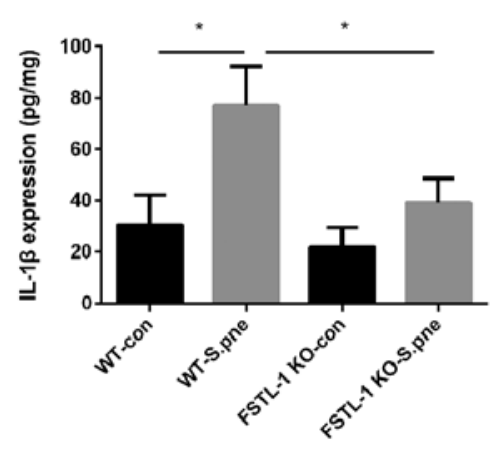

D

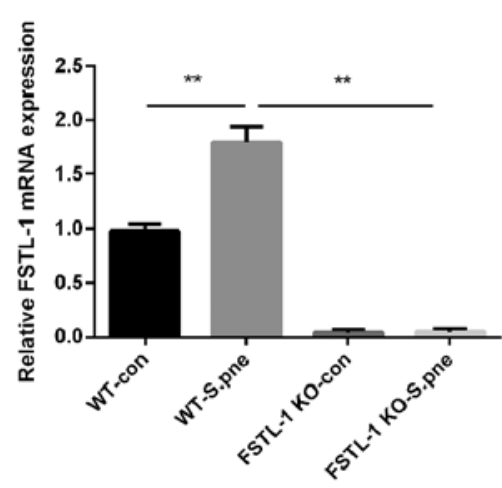

B

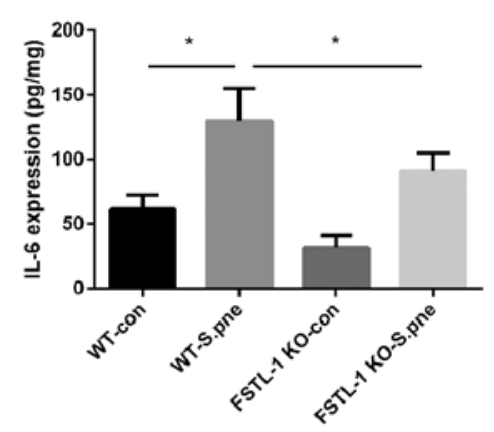

C

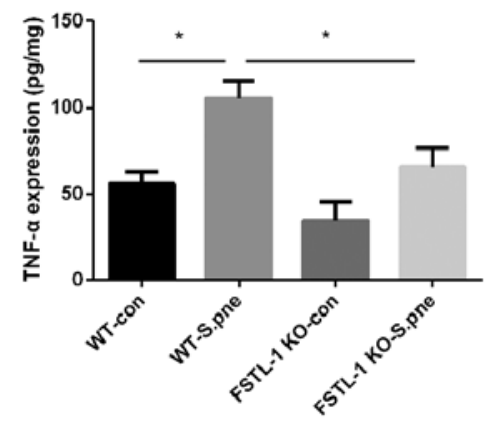

Figure 5. Knockout of FSTL-1 inhibits the inflammatory response against $S$. pneumoniae infection in vivo by suppressing the NLRP3 and TLR4/NF- $\mathrm{BB}$ signaling pathway. (A) IL-1 $\beta$ (B) IL-6, and (C) TNF- $\alpha$ expression levels were determined in bronchoalveolar lavage fluids of FSTL-1 KO mice infected with S. pne. (D) Protein levels of FSTL-1, NLRP3, TLR4, p-p65 were determined in lung tissues of FSTL-1 KO mice infected with S. pne (n=6). Similar results were obtained from at least three independent experiments. Data are expressed as the mean \pm SD. ${ }^{*} \mathrm{P}<0.05$; ${ }^{* *} \mathrm{P}<0.01$. FSTL-1, Follistatin-like protein 1 ; WT, wild-type; KO, knockout; IL, interleukin; TNF- $\alpha$, tumor necrosis factor- $\alpha$; S.pne, Streptococcus pneumoniae; con, control; NLRP3, nucleotide oligomerization domain-like receptor protein 3; TLR4, Toll-like receptor 4; p-, phosphorylated.

Knockdown of FSTL-1 protects against S.pneumoniae-induced inflammatory injury by inhibiting NLRP3 and through the TLR4/NF- $\kappa B$ signaling pathway in vivo. The present study found that FSTL-1 was important for inflammation in BMDMs infected with $S$. pneumoniae. Therefore, the role of FSTL-1 during $S$. pneumoniae infection in vivo was investigated. As shown in Fig. $5 \mathrm{~A}-\mathrm{C}$, the production of IL-1 $\beta$, IL- 6 and TNF- $\alpha$ in BALFs was increased significantly after C57BL/6 mice were infected with $S$. pneumoniae, and knockout of FSTL-1 decreased the expression of these factors. In addition, the mRNA expression of FSTL-1 detected by RT-qPCR and the protein levels of NLRP3, TLR4, p-p65 measured by western blotting in the lung tissues of infected FSTL-1 KO mice were decreased compared with the infected WT group (Fig. 5D). These results indicated that the knockout of FSTL-1 might protect against inflammatory injury induced by $S$. pneumoniae infection by inhibiting the NLRP3 and TLR4/NF- $\kappa$ B signaling pathway in vivo.

\section{Discussion}

In the present study, it was found that the infection of BMDMs with $S$. pneumoniae stimulated the expression of FSTL-1. Downregulation of FSTL-1 protected against inflammatory injury during $S$. pneumoniae infection by inhibiting NLRP3. In addition, it was found that the TLR4/NF- $\mathrm{B}$ signaling pathway was involved in inflammatory injury regulated by FSTL-1.

$S$. pneumoniae is a gram-positive bacterium that is a common cause of pneumonia. S. pneumoniae-mediated disease progression is significantly affected by the proinflammatory response during acute infection (21-23). Previous studies have shown that inflammatory cytokines were increased in the lung tissues during $S$. pneumoniae infection. For instance, Madouri et al (21) showed that IL-20, IL-1 $\beta$ and IL-6 cytokines were increased significantly during $S$. pneumoniae infection. Additionally, Li et al (24) showed that the expression levels of TNF- $\alpha$, IL- 6 and IL- $1 \beta$ in severe pneumonia induced by $S$. pneumoniae were increased significantly in vitro and in vivo. In the present study, it was found that the production of TNF- $\alpha$, IL- 6 and IL- $1 \beta$ was significantly increased in BMDMs infected with $S$. pneumoniae.

FSTL-1 plays an important role in the inflammatory response. For instance, Campfield et al (25) showed that FSTL-1 modulated IL-17-driven inflammation in stromal cells. Chaly et al (13) suggested that FSTL-1 was upregulated in patients with bacterial sepsis, and promoted the secretion of NLRP3 and IL-1 $\beta$ from monocytes and macrophages. The present study found that FSTL-1 was upregulated in BMDMs after S. pneumoniae injection. The overexpression of FSTL-1 promoted, while the knockdown of FSTL-1 suppressed the production of inflammatory factors. Moreover, it was also 
found that FSTL-1 positively modulated NLRP3. The present study suggested that the reduced expression of FSTL-1 may attenuate inflammatory injury during $S$. pneumoniae infection by inhibiting NLRP3.

NLRP3 was shown to be important in the inflammatory response (26). A previous study reported that in a meningitis model infected with $S$. pneumoniae, NLRP3 promoted an increase in host pathology (27). Stout-Delgado et al (28) found that macrophages infected with $S$. pneumoniae led to the development of harmful NLRP3-mediated inflammation and pulmonary fibrosis. Furthermore, excessive NLRP3 activation induced by $S$. pneumoniae infection was reported to induce an increase in the number of macrophages, which in turn increased morbidity (29). In the present study, it was found that NLRP3 was upregulated in BMDMs after S. pneumoniae injection and that depleting NLRP3 suppressed the production of IL- $1 \beta$, TNF- $\alpha$ and IL- 6 .

FSTL-1 has been reported to be induced by innate immune signals, including TLR4 agonists (30). TLR-induced FSTL-1 expression provides further understanding of the molecular mechanism of FSTL-1. Several previous studies have reported that TLR signaling may lead to the activation of NF- $\kappa \mathrm{B}(31-33)$. TLR4/NF- $\kappa$ B signaling was shown to be important for the immune response. For example, TLRs play an important role in IL- $1 \beta$ secretion and TLR $4 / \mathrm{NF}-\kappa \mathrm{B}$ signaling promoted the expression of IL-1 $\beta(34,35)$. In the present study, it was found that FSTL-1 modulated the TLR4/NF- $\kappa \mathrm{B}$ signaling pathway in vitro.

In conclusion, the present study demonstrated that the downregulation of FSTL-1 attenuated inflammation injury during $S$. pneumoniae infection by regulating NLRP3 in vitro and in vivo. The TLR4/NF- $\mathrm{B}$ signaling pathway was found to be involved in the inflammatory response during $S$. pneumoniae infection and was regulated by FSTL-1. However, there were limitations to the present study; for example, evidence was not provided to show the relationship between FSTL-1 and NLRP3. In the future, hematoxylin and eosin staining should be performed in lung tissues to investigate the injury levels modulated by FSTL-1, which could better demonstrate the effect of FSTL-1 on inflammatory injury.

\section{Acknowledgements}

Not applicable.

\section{Funding}

No funding was received.

\section{Availability of data and materials}

The datasets used and/or analyzed during the current study are available from the corresponding author on reasonable request.

\section{Authors' contributions}

ZL was a major contributor to the writing the manuscript and contributed to the conception of the study. LC performed the data analyses and wrote the manuscript. All authors read and approved the final study.

\section{Ethics approval and consent to participate}

The present study was approved by the Ethics Committee of Xian Yang Central Hospital.

\section{Patient consent for publication}

Not applicable.

\section{Competing interests}

The authors declare that they have no competing interests.

\section{References}

1. Rendon A, Rendon-Ramirez EJ and Rosas-Taraco AG: Relevant cytokines in the management of community-acquired pneumonia. Curr Infect Dis Rep 18: 10, 2016.

2. Adler A, Baraniak A, Izdebski R, Fiett J, Gniadkowski M, Hryniewicz W, Salvia A, Rossini A, Goossens H, Malhotra S, et al: A binational cohort study of intestinal colonization with extended-spectrum $\beta$-lactamase-producing proteus mirabilis in patients admitted to rehabilitation centres. Clin Microbiol Infect 19: E51-E58, 2013.

3. Domon H, Nagai K, Maekawa T, Oda M, Yonezawa D, Takeda W, Hiyoshi T, Tamura H, Yamaguchi M, Kawabata S and Terao Y: Neutrophil elastase subverts the immune response by cleaving toll-like receptors and cytokines in pneumococcal pneumonia. Front Immunol 9: 732, 2018.

4. Baldo V, Cocchio S, Gallo T, Furlan P, Romor P, Bertoncello C, Buja A and Baldovin T: Pneumococcal conjugated vaccine reduces the high mortality for community-acquired pneumonia in the elderly: An italian regional experience. PLoS One 11: e0166637, 2016.

5. Weycker D, Farkouh RA, Strutton DR, Edelsberg J, Shea KM and Pelton SI: Rates and costs of invasive pneumococcal disease and pneumonia in persons with underlying medical conditions. BMC Health Serv Res 16: 182, 2016.

6. Maus U, Rosseau S, Knies U, Seeger W and Lohmeyer J: Expression of pro-inflammatory cytokines by flow-sorted alveolar macrophages in severe pneumonia. Eur Respir J 11: 534-541, 1998.

7. Perny M, Roccio M, Grandgirard D, Solyga M, Senn P and Leib SL: The severity of infection determines the localization of damage and extent of sensorineural hearing loss in experimental pneumococcal meningitis. J Neurosci 36: 7740-7749, 2016.

8. Weichelt U, Cay R, Schmitz T, Strauss E, Sifringer M, Buhrer C and Endesfelder S: Prevention of hyperoxia-mediated pulmonary inflammation in neonatal rats by caffeine. Eur Respir J 41: 966-973, 2013.

9. Song C, Li H, Zhang Y and Yu J: Effects of Pseudomonas aeruginosa and Streptococcus mitis mixed infection on TLR4-mediated immune response in acute pneumonia mouse model. BMC Microbiol 17: 82, 2017.

10. Oshima Y, Ouchi N, Sato K, Izumiya Y, Pimentel DR and Walsh K: Follistatin-like 1 is an Akt-regulated cardioprotective factor that is secreted by the heart. Circulation 117: 3099-3108, 2008.

11. Wilson DC, Marinov AD, Blair HC, Bushnell DS, Thompson SD, Chaly Y and Hirsch R: Follistatin-like protein 1 is a mesenchyme-derived inflammatory protein and may represent a biomarker for systemic-onset juvenile rheumatoid arthritis. Arthritis Rheum 62: 2510-2516, 2010.

12. Miyamae T, Marinov AD, Sowders D, Wilson DC, Devlin J, Boudreau R, Robbins P and Hirsch R: Follistatin-like protein-1 is a novel proinflammatory molecule. J Immunol 177: 4758-4762, 2006.

13. Chaly Y, Fu Y, Marinov A, Hostager B, Yan W, Campfield B, Kellum JA, Bushnell D, Wang Y, Vockley J and Hirsch R: Follistatin-like protein 1 enhances NLRP3 inflammasome-mediated IL-1 $\beta$ secretion from monocytes and macrophages. Eur J Immunol 44: 1467-1479, 2014.

14. Katsuyama E, Miyamoto H, Kobayashi T, Sato Y, Hao W, Kanagawa H, Fujie A, Tando T, Watanabe R, Morita M, et al: Interleukin-1 receptor-associated kinase-4 (IRAK4) promotes inflammatory osteolysis by activating osteoclasts and inhibiting formation of foreign body giant cells. J Biol Chem 290: 716-726, 2015. 
15. Dinarello CA: Immunological and inflammatory functions of the interleukin-1 family. Annu Rev Immunol 27: 519-550, 2009.

16. Baroja-Mazo A, Martin-Sanchez F, Gomez AI, Martinez CM, Amores-Iniesta J, Compan V, Barbera-Cremades M, Yague J, Ruiz-Ortiz E, Anton J, et al: The NLRP3 inflammasome is released as a particulate danger signal that amplifies the inflammatory response. Nat Immunol 15: 738-748, 2014.

17. Medzhitov R: Toll-like receptors and innate immunity. Nat Rev Immunol 1: 135-145, 2001.

18. Kawai T and Akira S: The role of pattern-recognition receptors in innate immunity: Update on Toll-like receptors. Nat Immunol 11: 373-384, 2010.

19. Inaba K, Inaba M, Romani N, Aya H, Deguchi M, Ikehara S, Muramatsu S and Steinman RM: Generation of large numbers of dendritic cells from mouse bone marrow cultures supplemented with granulocyte/macrophage colony-stimulating factor. J Exp Med 176: 1693-1702, 1992.

20. Livak KJ and Schmittgen TD: Analysis of relative gene expression data using real-time quantitative PCR and the 2(-Delta Delta $\mathrm{C}(\mathrm{T})$ ) method. Methods 25: 402-408, 2001.

21. Madouri F, Barada O, Kervoaze G, Trottein F, Pichavant M and Gosset P: Production of interleukin-20 cytokines limits bacterial clearance and lung inflammation during infection by Streptococcus pneumoniae. EBioMedicine 37: 417-427, 2018.

22. Kato H, Yamagishi Y, Hagihara M, Yokoyama Y, Suematsu H, Asai N, Koizumi Y and Mikamo H: Antimicrobial activity of solithromycin and levofloxacin against a murine pneumonia mixed-infection model caused by Streptococcus pneumoniae and anaerobic bacteria. J Infect Chemother 25: 311-313, 2019.

23. Hsu CF, Hsiao CH, Tseng SF, Chen JR, Liao YJ, Chen SJ, Lin CS Sytwu HK and Chuang YP: PrtA immunization fails to protect against pulmonary and invasive infection by Streptococcus pneumoniae. Respir Res 19: 187, 2018.

24. Li H, Chen $X$ and Zhou SJ: Dauricine combined with clindamycin inhibits severe pneumonia co-infected by influenza virus H5N1 and Streptococcus pneumoniae in vitro and in vivo through NF-кB signaling pathway. J Pharmacol Sci 137: 12-19, 2018.

25. Campfield BT, Eddens T, Henkel M, Majewski M, Horne W, Chaly Y, Gaffen SL, Hirsch R and Kolls JK: Follistatin-like protein 1 modulates IL-17 signaling via IL-17RC regulation in stromal cells. Immunol Cell Biol 95: 656-665, 2017.
26. McElvaney OJ, Zaslona Z, Becker-Flegler K, PalssonMcDermott EM, Boland F, Gunaratnam C, Gulbins E, O'Neill LA, Reeves EP and McElvaney NG: Specific inhibition of the NLRP3 inflammasome as an anti-inflammatory strategy in cystic fibrosis. Am J Respir Crit Care Med: Aug 27, 2019 (Epub ahead of print).

27. Hoegen T, Tremel N, Klein M, Angele B, Wagner H, Kirschning C, Pfister HW, Fontana A, Hammerschmidt S and Koedel U: The NLRP3 inflammasome contributes to brain injury in pneumococcal meningitis and is activated through ATP-dependent lysosomal cathepsin B release. J Immunol 187: 5440-5451, 2011.

28. Stout-Delgado HW, Cho SJ, Chu SG, Mitzel DN, Villalba J, El-Chemaly S, Ryter SW, Choi AM and Rosas IO: Age-dependent susceptibility to pulmonary fibrosis is associated with NLRP3 inflammasome activation. Am J Respir Cell Mol Biol 55: 252-263, 2016.

29. Moon JS, Nakahira K, Chung KP, DeNicola GM, Koo MJ, Pabon MA, Rooney KT, Yoon JH, Ryter SW, Stout-Delgado H and Choi AM: NOX4-dependent fatty acid oxidation promotes NLRP3 inflammasome activation in macrophages. Nat Med 22: 1002-1012, 2016.

30. Clutter SD, Wilson DC, Marinov AD and Hirsch R: Follistatin-like protein 1 promotes arthritis by up-regulating IFN-gamma. J Immunol 182: 234-239, 2009.

31. Dong D, Zhou H, Na SY, Niedra R, Peng Y, Wang H, Seed B and Zhou GL: GPR108, an NF- $\kappa$ B activator suppressed by TIRAP, negatively regulates TLR-triggered immune responses. PLoS One 13: e0205303, 2018.

32. Liu L, Pang XL, Shang WJ, Xie HC, Wang JX and Feng GW: Over-expressed microRNA-181a reduces glomerular sclerosis and renal tubular epithelial injury in rats with chronic kidney disease via down-regulation of the TLR/NF- $\kappa \mathrm{B}$ pathway by binding to CRY1. Mol Med 24: 49, 2018.

33. Niu W, Sun B, Li M, Cui J, Huang J and Zhang L: TLR-4/ microRNA-125a/NF- $\mathrm{B}$ signaling modulates the immune response to Mycobacterium tuberculosis infection. Cell Cycle 17: 1931-1945, 2018.

34. Becker CE and O'Neill LA: Inflammasomes in inflammatory disorders: The role of TLRs and their interactions with NLRs. Semin Immunopathol 29: 239-248, 2007.

35. Bankers-Fulbright JL, Kalli KR and McKean DJ: Interleukin-1 signal transduction. Life Sci 59: 61-83, 1996. 